\title{
Think It Over
}

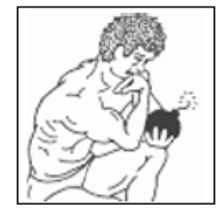

This section of Resonance presents thought-provoking questions, and discusses answers a few months later. Readers are invited to send new questions, solutions to old ones and comments, to 'Think It Over', Resonance, Indian Academy of Sciences, Bangalore 560080 . Items illustrating ideas and concepts will generally be chosen.

\section{An Interesting Problem on Diophantine Triple}

\section{Solution:}

We want to find three integers $a, b, c$ with $1 \leq a \leq$ $12,1 \leq b \leq 31,1 \leq c \leq 99$ such that $a b+1, a c+1$ and $b c+1$ are perfect squares. We desire ' $c$ ' to have the largest possible value since we want the last day of the century.

It is well known that the general form of a Diophantine triple is $(a, b, a+b \pm 2 x)$, where $x^{2}=a b+1$. As we want $c$ to be large, we should choose the ' + ' sign in the third term of the above triplet. The maximum possible value of $a b$ as per our conditions is (12) $(31)=372$. Now the largest square less than 373 is 361 . Hence we get $a=12, b=30$ and the corresponding value of $x$ as 19 which gives $a+b+2 x$ as 80 .

Thus the required date is $12 / 30 / 80$.

Remark. For each choice of $a, b$ where $a b+1=x^{2}$, there is another choice of $c$ other than $a+b \pm 2 x$ which is given by $4 x(a+x)(b+x)$. But this choice is not to be considered as we want $c \leq 99$.
K S Bhanu ${ }^{1}$ and M N Deshpande ${ }^{2}$

${ }^{1}$ Department of Statistics Institute of Science Nagpur 440 001, India 2 22, Panchdeep Nagar Wardha Road Nagpur 440 001, India.

Problem appeared in Resonance, Vol.19, No.2, p.190, 2014.

Keywords

Diophantine triple. 